\title{
Aumento da sobrevida de pacientes com cardiopatias congênitas após assistência perinatal e neonatal adequada: relato de caso
}

\author{
Increased survival of patients with congenital heart disease after perinatal and neonatal \\ care: case report
}

\begin{abstract}
Aumento de la supervivencia de pacientes con cardiopatia congénita después de uma atención perinatal y neonatal adecuada: reporte de caso
\end{abstract}

Taciana Carreira de Aquino ${ }^{1 *}$, Beatriz Britto Barufi', Patrícia Marques Mendes ${ }^{1}$, Taíssa Naves Araújo ${ }^{2}$, Kelly Luísa Cintra¹.

\begin{abstract}
RESUMO
Objetivo: Demonstrar o aumento da sobrevida dos pacientes com cardiopatias congênitas em condições desfavoráveis após adequada assistência neonatal e da correta indicação de interrupção do parto. Detalhamento do caso: Lactente, masculino, 7 meses de idade, paciente em um Ambulatório pediátrico em uma cidade do Estado de São Paulo. Paciente nasceu de 30 semanas e 3 dias de gestação, via parto cesárea por taquicardia fetal e diminuição do movimento fetal, pesando 1900 gramas, medindo 41 centímetros e com APGAR 3/6. Ao nascer, foi colocado em ventilação mecânica, apresentou depressão neonatal, síndrome de desconforto respiratório do recémnascido, taquicardia supraventricular, hidropsia fetal e hipoglicemia neonatal. Em ecocardiograma foi mostrado transposição congênita corrigida dos vasos da base (TCGA) e uma insuficiência tricúspide de grau leve. O eletrocardiograma revelou ritmo sinusal, frequencia cardiaca de $136 \mathrm{bpm}$, eixo a zero graus e intervalo PR curto evidenciando uma possível síndrome de Wolff-Parkinson-White. O Holter de 24h, exibiu 43 episódios de taquicardia juncional paroxística e raras extrassístoles. Considerações finais: A importância de uma triagem pós-natal adequada em relação às taquicardias e apnéias proporciona ao paciente um tratamento precoce, e consequentemente um prognóstico melhor do que se tivesse tido o diagnóstico na fase adulta, período que prevalece os diagnósticos de TCGA.
\end{abstract}

Palavras-chave: Cardiopatias congênitas, Transposição congênita corrigida de grandes artérias, Triagem neonatal.

\begin{abstract}
Objective: To demonstrate the increased survival of patients with congenital heart disease in unfavorable conditions after adequate neonatal care and correct indication on pregnancy interruption. Details of the case: Infant, male, 7 months years old, patient in a pediatric outpatient Clinic in a city in the state of São Paulo. The patient was born with 30 weeks and 3 days of gestation, delivered by cesarean due to fetal tachycardia and decreased fetal movements. Weighting 1900 grams, measuring 41 centimeters and APGAR of 3/6. At birth, he was placed on mechanical ventilation, had neonatal depression, newborn's respiratory distress syndrome, supraventricular tachycardia, fetal hydrops and neonatal hypoglycemia. An echocardiogram showed preserves systolic function of ventricles, congenitally corrected transposition of the basal vessels (CCTGA) and mild tricuspid insufficiency. An electrocardiogram revealed sinus rhythm, heart rate of $136 \mathrm{bpm}$, axis at zero degrees and short PR interval showing a possible Wolff-Parkinson-White Syndrome (WPW) while the 24-hour Holter exam showed 43 episode of tachycardia paroxysmal junction, rare extrasystoles. Final considerations: The importance of adequate postnatal screening in relation to tachicardias and apneas provides the patient with early treatment, and consequently a better prognosis than if he had the diagnosis in adulthood, a period that diagnoses of CCTGA prevails.
\end{abstract}

Keywords: Heart defects congenital, Congenitally corrected transposition of the great arteries, Neonatal screening.

\footnotetext{
${ }^{1}$ Universidade de Franca (UNIFRAN), Franca - SP. *E-mail: taci.med4@gmail.com
}

${ }^{2}$ Santa Casa de Misericórdia de Franca, Franca - SP.

SUBMETIDO EM: 8/2020

ACEITO EM: 9/2020

PUBLICADO EM: 10/2020 


\section{RESUMEN}

Objetivo: Demostrar el aumento de la supervivencia de pacientes con cardiopatía congénita en condiciones desfavorables después de una atención neonatal adecuada y la indicación correcta de interrupción del parto. Detalles del caso: bebé, hombre, 7 meses de edad, paciente en consulta externa de pediatría en una ciudad del estado de São Paulo. La paciente nació a las 30 semanas y 3 días de gestación, por cesárea debido a taquicardia fetal y disminución del movimiento fetal, con un peso de 1900 gramos, que mide 41 centímetros y con APGAR 3/6. Al nacer, fue colocado en ventilación mecánica, tenía depresión neonatal, síndrome de dificultad respiratoria del recién nacido, taquicardia supraventricular, hidropesía fetal e hipoglucemia neonatal. Un ecocardiograma mostró una transposición congénita corregida de los vasos base (TCGA) y una insuficiencia tricúspide leve. El electrocardiograma reveló ritmo sinusal, frecuencia cardíaca de $136 \mathrm{lpm}$, eje a cero grados e intervalo PR corto, mostrando un posible síndrome de Wolff-Parkinson-White. El Holter de 24 horas exhibió 43 episodios de taquicardia de unión paroxística y extrasístoles raras. Concideraciones finales: La importancia de un cribado posnatal adecuado en relación con las taquicardias y las apneas proporciona al paciente un tratamiento temprano y, en consecuencia, un mejor pronóstico que si hubiera tenido el diagnóstico en la edad adulta, un período en el que prevalecen los diagnósticos de TCGA.

Palabras clave: Cardiopatías congénitas, Transposición congénitamente corregida de las grandes, Tamizaje neonatal.

\section{INTRODUÇÃO}

Cardiopatias congênitas (CC) são caracterizadas como malformações anatômicas grosseiras do coração ou dos grandes vasos intratorácicos com evidente importância funcional (PINTO CP, et al., 2018). São responsáveis por $40 \%$ dos defeitos congênitos e trazem grande morbimortalidade para os recém-nascidos (MALIK AA, et al., 2019).

A transposição de grandes vasos (TGA) caracteriza-se por uma cardiopatia congênita cianótica ventrículo arterial discordante em que os vasos da base, artéria aorta e artéria pulmonar originam-se respectivamente no ventrículo direito e ventrículo esquerdo. Para sua "compatibilidade" com a vida é necessário a existência de shunt entre a circulação pulmonar e sistêmica, visto isso em cerca de $40 \%$ dos casos de TGA há abertura do septo interventricular (SWANSON SK, et al., 2018).

Esta patologia corresponde a cerca de $8 \%$ das cardiopatias congênitas existentes, sendo bem mais prevalente no sexo masculino (3:1) e de alta mortalidade, necessitando, na maioria dos casos, de conduta cirúrgica precoce, de preferência no período neonatal. Pode ser classificada em simples e complexa, na primeira o paciente pode apresentar cianose já ao nascimento e hiperfonese de segunda bulha cardíaca em foco tricúspide ou pulmonar. Já na forma complexa a cianose é mais discreta, no entanto, pode acarretar em insuficiência cardíaca em um mês devido ao alto fluxo pulmonar (LIMA TS, et al., 2016).

A transposição congênita corrigida de grandes artérias (TCGA) é uma anomalia cardíaca extremamente rara na qual existe, além de uma discordância ventrículo-arterial, uma discordância átrio-ventricular, em que o átrio direito ( $A D$ ) se comunica com o ventrículo esquerdo (VE) e vice-versa (LENOIR M, et al., 2018).

A discordância atrioventricular é uma cardiopatia congênita incomum, responsável por $0,05 \%$ das cardiopatias congênitas, e com prevalência aproximada de 0,07 a cada 1.000 nascidos vivos (LENOIR M, et al.,2018). Há discreta prevalência em meninos em relação as meninas $(1,6: 1)$ (SANTANA MVT, 2014).

Tal anomalia faz com que o sangue venoso caminhe do ventrículo esquerdo para a arvore pulmonar arterial. Além disso, as valvas atrioventriculares acompanham o devido ventrículo, sendo a mitral à direita $e$ tricúspide à esquerda. A repercussão clínica depende da gravidade das anomalias associadas, assim como da repercussão hemodinâmica causada pelas mesmas (KUTTY S, et al., 2018).

Esta patologia pode apresentar-se com situs solitus (L-TGA) ou com situs inverso (D-TGA). Na TCGA a sintomatologia é dependente da funcionalidade do ventrículo direito e das patologias associadas, sendo as mais frequentes a comunicação interventricular e os bloqueios átrio-ventriculares (SPIGEL Z, et al., 2019). 
A terapêutica para L-TGA envolve tratamento cirúrgico, podendo ser de forma paliativa ou definitiva a depender da condição do paciente. Deve-se levar em conta o número e a complexidade das alterações anatômicas, função do ventrículo direito, competência de tricúspide e outros. As cirurgias paliativas ainda tem grande importância terapêutica nos primeiros anos de vida, porém nos últimos anos a correção anatômica (duplo suíte) vem sendo cada vez mais estudada e aplicada na pratica medica, apesar de necessitarem mais dados a longo prazo (PENHA JG, et al., 2015).

Em média 2 em cada 1.000 recém-nascidos vivos apresentam algum tipo de cardiopatia congênita crítica e, 30\% destes recém-nascidos recebem alta hospitalar sem o diagnóstico, podendo evoluir com complicações graves (QUEIROZ A, et al., 2020).

Com o aumento da evolução tecnológica, vários dispositivos estão sendo incorporados na prática hospitalar para detecção precoce de patologias associadas ao sistema cardiovascular, como a oximetria de pulso (teste do coraçãozinho) que tem um papel importantíssimo na detecção de cardiopatias em neonatos (MEDEIROS AL, et al., 2015).

O teste do coraçãozinho associado ao exame físico apresenta-se como um método de triagem para o diagnóstico de cardiopatia congênita. A confirmação da cardiopatia será feita com a realização do ecocardiograma, será submetido ao exame, para confirmação de diagnóstico apenas aqueles neonatos que apresentarem alguma alteração no exame físico ou na oximetria de pulso (MEDEIROS AL, et al., 2015).

Em se tratando das CC, além da triagem neonatal, deve ser levado em conta os sinais pré-natais que levam o obstetra a sugerir uma possível patologia cardíaca e então interromper esta gestação. Um dos sinais precocemente observados é o sofrimento fetal agudo, definido como a presença de hipoxemia e hipercapnia advindo de trocas gasosas não eficazes, podendo ocorrer no período perinatal ou anteparto (PEDRA SRF, et al., 2019).

Algumas das causas podem ser de origem útero-placentária, sendo o principal exemplo a pré-eclâmpsia, ou feto-placentária, como a oligodramnia. Para que seja feito o diagnóstico de um sofrimento fetal agudo deve ser feita a avaliação fetal antenatal, empregada de forma rotineira em gestações de alto risco nas quais o risco de comprometimento fetal é sabidamente aumentado, com o intuito de identificar fetos que se beneficiariam de intervenções oportunas (SANTOS RCS, 2018).

Os métodos diagnósticos baseiam-se na prerrogativa de que o feto com hipoxemia apresentará quadro clínico compatível com respostas adaptativas fisiológicas, evoluindo para sinais de descompensação fetal. Uma delas é o mecanismo hemodinâmico fetal de compensação, que envolve a estimulação do sistema nervoso autônomo fetal, causando aumento da resistência vascular periférica e gradativo aumento da frequência cardíaca fetal (FCF).

Temos também como método diagnóstico o registro de movimentos fetais, sendo um dado de fácil observação, apesar de subjetivo e não comprobatório, pode ser investigado na anamnese inicial e está relacionado a anomalias congênitas, restrição de crescimento fetal, prematuridade e óbito perinatal. A movimentação fetal se reduz frente à hipoxemia, portanto, quando são relatadas mudanças abruptas no padrão de movimentação do concepto, elas podem revelar comprometimento fetal, exigindo investigação por propedêutica especializada, por exemplo cardiotocografia (OLIVEIRA CA e SÁ RAM, 2018).

Sendo assim, a interrupção da gravidez deve ser considerada uma vez que há consenso de que na presença de sofrimento fetal agudo real, a indicação de cesariana é necessária, sendo recomendada a realização da mesma no menor intervalo de tempo. A literatura tem demonstrado que um intervalo de 30 minutos parece ser adequado para a maioria dos casos (SILVA AP, et al., 2019).

Este relato de caso visa demonstrar a importância do aumento na sobrevida dos pacientes com cardiopatias congênitas mesmo em condições desfavoráveis, baixo peso e prematuridade após a adequada assistência neonatal da equipe multiprofissional, da correta indicação de interrupção do parto e do satisfatório seguimento em um ambulatório especializado. 


\section{DETALHAMENTO DO CASO}

Lactente do sexo masculino, 7 meses de idade, natural e procedente de uma cidade no interior do Estado de São Paulo, em seguimento em um Ambulatório Pediátrico da cardiopatia congênita. Paciente nasceu de 30 semanas e 3 dias de gestação, feto de mãe com deficiência auditiva por sequela de rubéola congênita, via parto cesárea por taquicardia fetal e diminuição de movimento fetal, com peso de nascimento de 1900 gramas, estatura de 41 centímetros, perímetro cefálico de 30 centímetros e escala de APGAR 3/6.

Ao nascer, o paciente foi colocado em ventilação mecânica, apresentou depressão neonatal de rápida recuperação, síndrome de desconforto respiratório do recém-nascido necessitando de uma dose de surfactante, taquicardia supraventricular revertida espontaneamente em poucos minutos, hidropsia fetal e hipoglicemia neonatal resolvida após uso de um push de glicose. Foi realizado eletrocardiograma que demonstrou ritmo sinusal e frequência cardíaca de 150 batimentos por minuto.

Foi feito ecocardiograma no segundo dia de vida do paciente, evidenciando uma função sistólica dos ventrículos preservada, transposição congênita corrigida dos vasos da base (TCGA) e uma insuficiência tricúspide de grau leve. No mesmo dia, foi realizado um novo eletrocardiograma que revelou ritmo sinusal, frequencia cardiaca de 136 batimentos por minuto, eixo a zero graus e intervalo entre as ondas PR curto evidenciando uma possível síndrome de Wolff-Parkinson-White (WPW). Já no exame Holter de 24h, foi apresentado 43 episódios de taquicardia juncional paroxística, raras extrassístoles e não sendo possível descartar a hipótese de síndrome WPW.

O paciente evoluiu com icterícia neonatal precoce, corrigida com fototerapia por 6 dias, além de outros episódios de taquicardia supraventricular revertidos com uma dose de Adenosina ( $0,1 \mathrm{mg} / \mathrm{kg} / \mathrm{dose})$, seguido de outra dose de $0,15 \mathrm{mg} / \mathrm{kg} / \mathrm{dose}$, além de dose ataque de Amiodarona $(5 \mathrm{mg} / \mathrm{kg})$, seguindo para uso de manutenção de Amiodarona (15 mg/kg/dia). Também apresentou distúrbios hidroeletrolíticos sendo resolvidos durante o período de internação. Evoluiu com apneia da prematuridade após extubação, com episódios de apneia resolvidos com uso de Continuous Positive Airway Pressure (CPAP) e cateter nasal com suporte de oxigênio, e episódios de apneia mais prolongada e associada a cianose importante e queda da saturação de oxigênio revertidos com estimulação tátil e aumento do volume de oxigênio de suporte.

Mesmo com a progressão adequada da dieta, o recém-nascido evoluiu com distensão abdominal devido a alergia a proteína do leite e obstrução intestinal, necessitando de um estímulo retal para melhora do quadro intestinal. Durante a internação, também apresentou edema generalizado associado a episódios de engasgos após mamadas e queda da saturação de oxigênio e aparecimento de cianose em face, mantendo dispneia leve e alguns períodos de taquipneia, tendo o quadro aliviado com uso de furosemida $(0,5 \mathrm{mg} / \mathrm{kg}) \mathrm{como}$ manutenção.

Após a alta hospitalar, paciente continuou seguimento no ACAR, onde sua responsável (mãe) relatou evolução com dispneia durante a amamentação, associada a sudorese. Mãe relata ter cianose em face apenas ao fazer esforço para evacuar e que apresentou um episódio de hipotonia, como se tivesse desmaiado, mas retornou ao normal logo e espontâneo.

Paciente faz uso da fórmula Neocate $55 \mathrm{ml}$ de $3 / 3 \mathrm{~h}$ ( $132 \%$ de leite e $24 \%$ de água = total de $156 \%$ ). Mãe relatou que em algumas vezes o lactente não consegue terminar toda a mamadeira por conta da dispneia e que mantém uma ingesta hídrica de alguns mililítros por dia.

Paciente teve bom ganho de peso, apresentou desenvolvimento neuropsicomotor normal, expansibilidade torácica com uso de musculatura acessória, taquipneia e respiração em salvas. Ao exame físico pulmonar apresentou murmúrio vesicular fisiológico bilateral, frêmito toracovocal presente e som claro pulmonar em percussão torácica. À ausculta cardíaca demonstrou frequencia cardíaca normal, com duas bulhas normofonéticas rítmicas com sopro 2+/6+ em borda esternal inferior esquerda. Em ausculta pulmonar estava taquipneico, com murmúrio vesicular fisiológico globalmente e sem presença de ruídos adventícios.

Paciente segue em uso de Furosemida $40 \mathrm{mg}$, Amiodarona 200mg Ranitidina 15mg/ml, Domperidona $1 \mathrm{mg} / \mathrm{ml}$ Sulfato Ferroso 12gts e Polivitamínico 12gts ao dia. A responsável pelo paciente foi orientada quanto a importância de restrição hídrica e a necessidade de aumento da medicação para otimizar a pré-carga. 


\section{DISCUSSÃO}

O relato traz uma ocorrência de discordância atrioventricular, também conhecida como transposição congenitamente corrigida das grandes artérias (TCGA), e caracterizada por uma patologia onde há conexão entre o átrio esquerdo com o ventrículo direito e o átrio direito com o ventrículo esquerdo e concomitantemente por uma união entre artéria pulmonar e ventrículo esquerdo e aorta com ventrículo direito (SANTANA MVT, 2014). Sendo diagnosticado precocemente logo após seu nascimento, decorrente de um parto cesareano de urgência por taquicardia fetal e manutenção de episódios de taquicardia e apneias pós-natal.

Destaca-se a importância de uma triagem pós-natal adequada em relação às taquicardias e apnéias, uma vez que , com o tratamento precoce e adequado, é possível oferecer um melhor prognóstico ao paciente do que se tivesse tido um diagnóstico entre seus 20 a 40 anos, faixa etária mais prevalente dos diagnósticos de TCGA. Assim, podemos estar a frente do aparecimento de lesões frequentemente relacionadas à discordância atrioventricular como a comunicação interventricular (CIV) e a estenose pulmonar, encontrados em cerca de $80 \%$ dos casos, além da insuficiência tricúspide, sendo as alterações na valva tricúspide responsáveis por disfunções em ate $90 \%$ dos casos (DAY TG, et al., 2019).

As manifestações clínicas relacionadas à presença de TCGA são, em geral, de insuficiência tricúspide, sendo cianose e dispneia os sintomas mais comuns. Com o início de seguimento e tratamento nos primeiros anos de vida, pode-se evitar, ou ao menos adiar, o desenvolvimento com o passar dos anos de um bloqueio atrioventricular total, o qual necessitaria de implante de marcapasso. Adiando também uma possivel evolução com disfunção sistólica do ventrículo esquerdo e insuficiência aórtica.

\section{CONSIDERAÇÕES FINAIS}

Conclui-se que apesar da alta taxa de morbimortalidade da patologia discutida, demonstou-se que com a intervenção adequada e precoce combinada a um seguimento interdisciplinar durante a puerilcultura podemos ter um aumento significativo da sobrevida desta criança. Desta forma, destaca-se a importância de se realizar um diagnóstico precoce, o qual atualmente pode ser feito ainda na fase gestacional, através da ecografia fetal, ou no período pós-natal, por meio do exame de ecocardiografia, com a intenção de que o tratamento clínico e/ou cirúrgico mais efetivo seja instituído em tempo hábil, modificando dessa forma a história natural da doença e melhorando o prognóstico do paciente. Além disso reitaramos a particularidade de cada paciente levando a uma logistica de tratamento individualizado a depender das possiveis combinações de patologias que normalmente podem estar associadas em casos de cardiopatias congênitas.

\section{REFERÊNCIAS}

1. DAY TG, et al. Postnatal Outcome Following Prenatal Diagnosis of Discordant Atrioventricular and Ventriculoarterial Connections. Pediatric Cardiology. 2019; 40(7):1509-1515

2. KUTTY S, et al. Contemporary management and outcomes in congenitally corrected transposition of the great arteries. Heart, 2018; 104(14): 1148-1155.

3. LENOIR M, et al. Outcomes of the anatomical repair in patients with congenitally corrected transposition of the great arteries: lessons learned in a high-volume centre. European Journal of Cardio-Thoracic Surgery. 2018; 54(3): 532538.

4. LIMA TS, et al. Transposition of great vessels: a mapping of the Brazilian literature. Rev de Enfermagem UFPE. 2016; 10: $52-62$.

5. MALIK AA, et al. Agreement Between 64-Slice Multidetector CT Angiography and Transthoracic Echocardiography in Detection of Extracardiac Findings of Congenital Heart Disease. J Coll Physicians Surg Pak, 2019; 29(10): 923-927.

6. MEDEIROS AL, et al. Oximetria de pulso em triagem de cardiopatias congênitas: conhecimento e atuação do enfermeiro. Cogitare enferm, 2015; 20(3): 605-611.

7. OLIVEIRA CA, SÁ RAM. Cardiotocogra a anteparto. Federação Brasileira das Associações de Ginecologia e Obstetrícia (FEBRASGO), 2018: Protocolo FEBRASGO - Obstetrícia, no. 81/Comissão Nacional Especializada em Medicina Fetal.

8. PEDRA SRF, et al. Diretriz Brasileira de Cardiologia Fetal. Arq. Bras. Cardiol, 2019; 112(5):600-648.

9. PENHA JG, et al. Senning paliativo no tratamento de cardiopatias congênitas com hipertensão pulmonar grave. Arq. Bras. Cardiol, 2015: 105(4): 353-361. 
10. PINTO CP, et al. Fatores de riscos materno associados à cardiopatia congênita. J Health Sci Inst 2018: 36(1): 34-08.

11. QUEIROZ A, et al. A importância do teste do coraçãozinho no diagnóstico precoce de cardiopatias congênitas. Revista Científica de Enfermagem, 2020; 10(29): 145-154.

12. SANTANA MVT. Cardiopatias congênitas no recém-nascido: diagnóstico e tratamento. 3 ed. São Paulo: Atheneu, 2014: 588p.

13. SANTOS RCS. Parto cesariano em um centro obstétrico de referência no Estado do Amapá: estudo transversal. Estação Científica (UNIFAP), 2018; 8(2): 103-110.

14. SILVA AP, et al. As indicações de cesáreas no Brasil: Uma revisão de literatura integrativa. Revista Eletrônica Acervo Saúde, 2019; 24: e624.

15. SPIGEL Z, et al. Congenitally Corrected Transposition of the Great Arteries: Anatomic, Physiologic Repair, and Palliation. Semin Thorac Cardiovasc Surg Pediatr Card Surg Annu., 2019; 22: 32-42.

16. SWANSON SK, et al. Interpretation and Reporting of Coronary Arteries in Transposition of the Great Arteries. Journal of Thoracic Imaging.,2018; 33(4): 14-21. 